Case Report

\title{
Mucor Mycosis in COVID- I9: Case Reports
}

\author{
Ranjana Deshmukh', Kavita Upadhyay', Ravindra Patwadkar', Shridhan Patil $^{4}$ \\ ${ }^{1}$ Consultant and Head, Department of Medicine, Dr. Hedgewar Hospital, Aurangabad, Maharashtra, India. \\ ${ }^{2}$ Resident, DNB General Medicine, Dr. Hedgewar Hospital, Aurangabad, Maharashtra, India. \\ ${ }^{3} \mathrm{HOD}$ of Pathology Dept., Dr. Hedgewar Hospital, Aurangabad, Maharashtra, India. \\ ${ }^{4}$ Consultant Pathologist, Dr. Hedgewar Hospital, Aurangabad, Maharashtra, India. \\ DOI: https://doi.org/10.24321/2349.7181.202016
}

I $\quad \mathbf{N} \quad \mathbf{F} \quad \mathbf{O}$

\section{Corresponding Author:}

Kavita Upadhyay, Department of medicine, Dr. Hedgewar Hospital, Aurangabad, Maharashtra, India.

E-mail Id:

kavita3194@rediffmail.com

Orcid Id:

https://orcid.org/0000-0002-7111-6671

How to cite this article:

Deshmukh R, Upadhyay K, Patwadkar R. Patil S. Mucor Mycosis in COVID-19: Case Reports. J Adv Res Med 2020; 7(3): 20-23.

Date of Submission: 2020-12-02

Date of Acceptance: 2021-01-04

\section{$\begin{array}{llllllll}\mathbf{A} & \mathbf{B} & \mathbf{S} & \mathbf{T} & \mathbf{R} & \mathbf{A} & \mathbf{C} & \mathbf{T}\end{array}$}

Coronavirus infections are associated with a wide range of bacterial and fungal co-infections. Use of steroids, monoclonal antibodies and broad spectrum antibiotics along with underlying pathogenesis may alter body homeostasis and exacerbate preexisting fungal disease. We report the cases with COVID-19 infection, which, after the course of the treatment, presented with various forms of mucormycosis infection.

Keywords: Mucormycosis, COVID-19, Fungal Infections

\section{Introduction}

Coronavirus infection is associated with a broad array of disease patterns. Various bacterial and fungal co-infections may exist and may be exacerbated due to preexisting morbidity (like diabetes mellitus). India, being a developing nation has undergone increase in prevalence of diabetes mellitus and various lifestyle disorders. We report the cases of COVID-19 infection, with underlying diabetes which further presented with mucormycosis of various paranasal sinuses.

\section{Case Presentation}

\section{Case I}

A 39 year old diabetic female, presented with pain and swelling around left orbital and maxillary area since 3 days associated with fever and anorexia. On admission she was febrile with pulse rate of 110 beats/ $\mathrm{min}$. She underwent routine laboratory investigation with radiological imaging
(Table 1). poor glycemic control was noted at presentation. $\mathrm{CT}$ as well as MRI Brain \& PNS revealed Heterogenous e nhancing T1 intense and T2 FLAIR heterogeneously hyper intense soft tissue noted in left maxillary sinus, left ethmoidal air cells and left nasal cavity extending posteriorly upto anterior portion of left pterygopalatine fossa superiorly up to cribriform plate and inferior part of frontal sinus. Similar small heterogenously enhancing soft tissue also noted in right ethmoid air cells and right nasal cavity. No intracranial extension was noted. These findings were suggestive of an invasive fungal sinusitis involving bilateral ethmoid air cells (Figures 1 and 2). Presence of fever in this pandemic era demanded further investigation for COVID 19 infection. COVID19 RTPCR as well as rapid antigen test was negative and SARS CoV 2 IgG antibody was positive. This suggests that there may have been prior asymptomatic COVID19 infection. Managed with I.V Amphotericin B 50mg. for 21 days. 


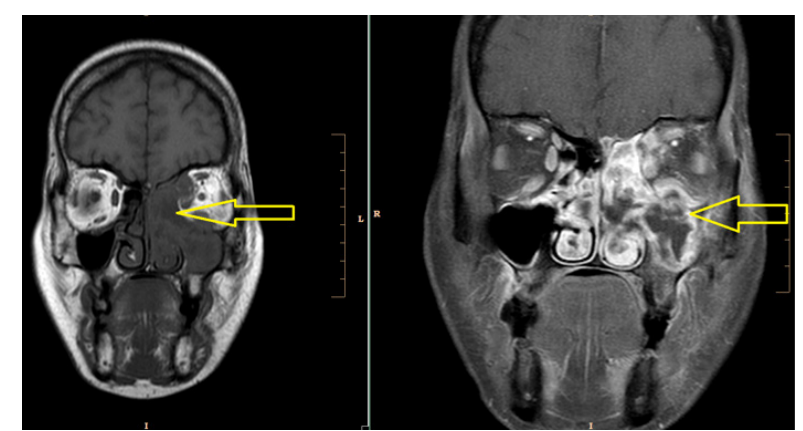

Figure I.MRI PNS Coronal view (left - non-contrast, right - post contrast films)

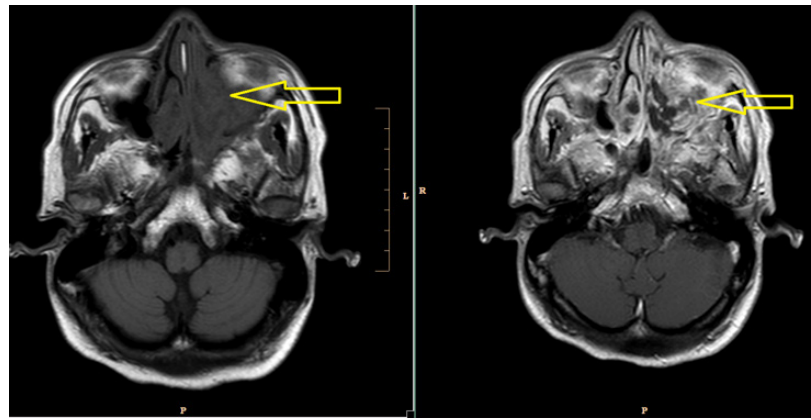

Figure 2.MRI PNS Axial view (left - non-contrast, right - post contrast films)

Showing invasive fungal sinusitis involving bilateral ethmoid air cells (yellow arrows).

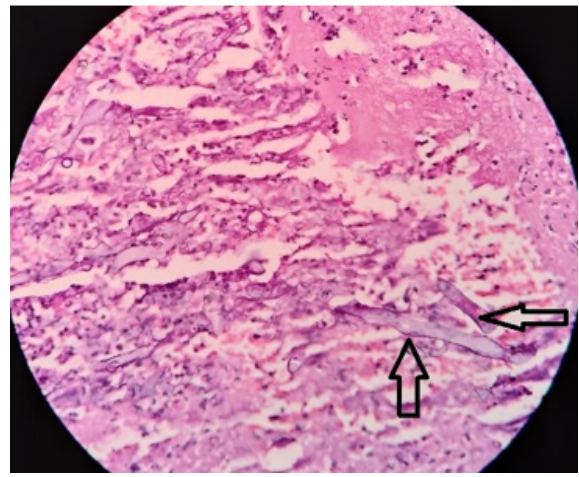

Figure 3.Histopathology of nasal scrapping demonstrating presence of Mucor (black arrow)

\section{Case 2}

A 43 year old diabetic male patient about 30 days after recovering from COVID-19 infection presented with pain over bilateral maxillary areas. He was hemodynamically stable during presentation. Routine laboratory investigations were performed (Table 1). Good glycemic control was noted. CT PNS reported maxillary fungal sinusitis. During the course of treatment for COVID infection he had received a course of low dose steroids along with supportive management. Total maxillectomy was planned. Histopathology specimen was suggestive of mucor mycosis. He was treated with amphotericin lyophilized (40mg) for 21 days. During the course his KFT was deranged, hence lyophilized form of amphotericin was preferred.

\section{Case 3}

A 50 year old diabetic male presented with persistent headache throat pain and anorexia since about 7 days. He had been treated for COVID-19 disease about 2 months ago during which he received appropriate dose of steroids. During presentation he was hemodynamically stable with poor glycemic control. Routine investigations are described in Table 1. On examination a lesion was noted in hard as well as soft palate. MRI PNS revealed possibility of fungal sinusitis with extension in infratemporal fossa on left side with changes of cellulitis in posterior aspect of left orbit (Figures 4\&5). He was managed conservatively amphotericin B 50mg. I.V daily for 21 days.

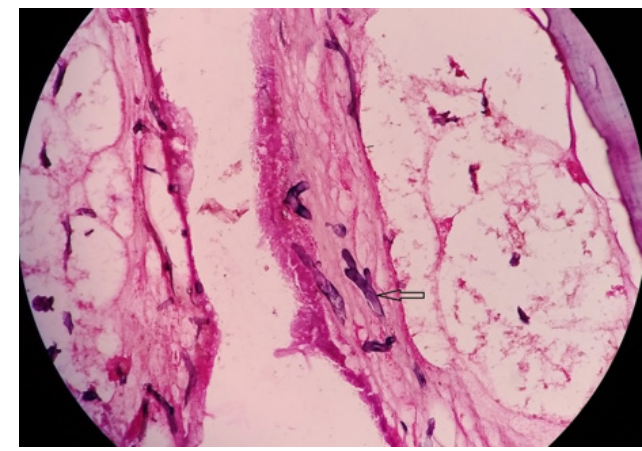

Figure 4. Please provide Caption

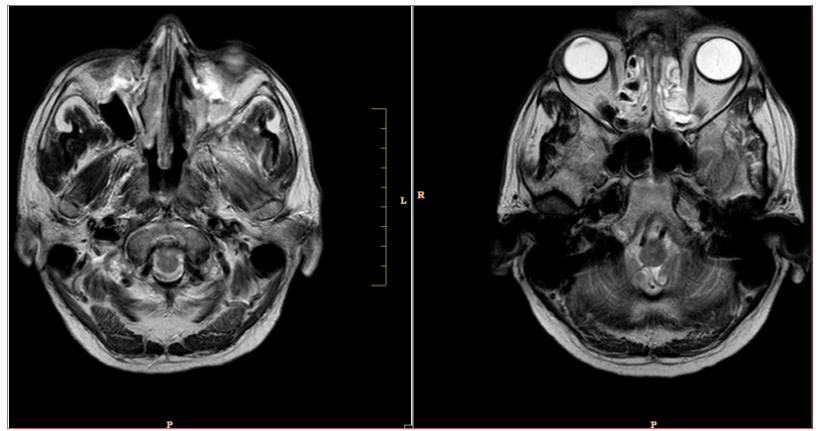

Figure 5.MRI PNS Axial view (left - non-contrast, right - post contrast)

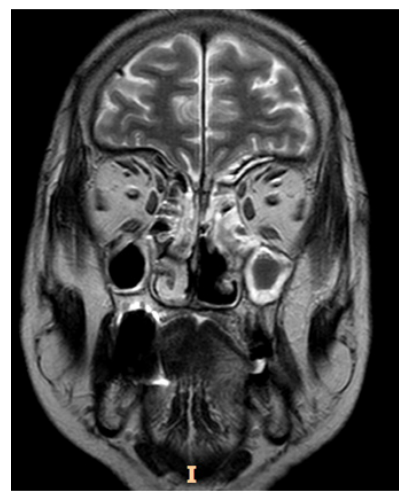

Figure 6.MRI PNS Coronal view, post contrast 


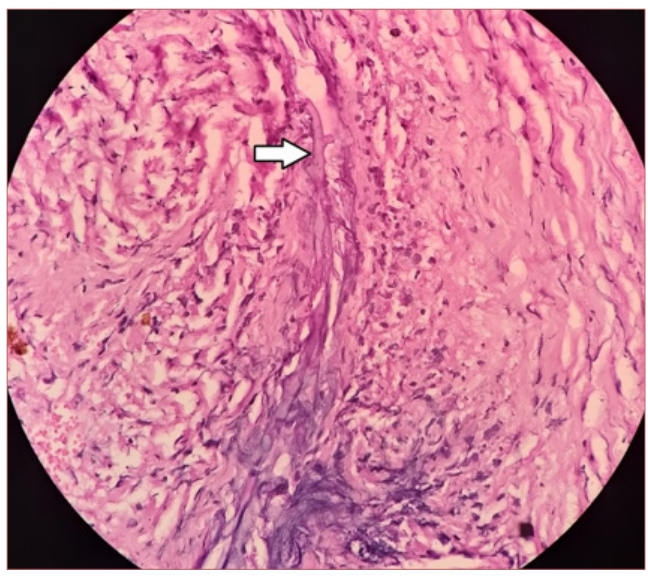

Figure 6. Histopathology of PNS showing mucor

Table I.Investigations of patient

\begin{tabular}{|c|c|c|c|}
\hline Investigations & Case 1 & Case 2 & Case 3 \\
\hline Hemoglobin g/ dl & 7.7 & 12.7 & 8 \\
\hline $\begin{array}{c}\text { Total leucocyte count } \\
\text { cells/ mm3 }\end{array}$ & 7800 & 8000 & 10200 \\
\hline Neutrophils \% & 65 & 69 & 53 \\
\hline Lymphocytes \% & 24 & 22 & 17 \\
\hline Creatine & 1.26 & 0.72 & 1.37 \\
\hline SGOT & 28 & 27 & 18 \\
\hline SGPT & 22 & 21 & 135 \\
\hline Na & 4.3 & 140 & 3.3 \\
\hline K & 548 & 4.3 & 280 \\
\hline $\begin{array}{c}\text { Random blood Glucose at } \\
\text { presentation mg/ dl }\end{array}$ & Negative & 124 & Negative \\
\hline SARS CoV 2 RTPCR & Negative & Negative & Negative \\
\hline SARS CoV 2 Rapid Antigen & Positive & Positive & Negative \\
\hline SARS CoV 2 IgG & $\begin{array}{c}\text { Invasive fungal sinusitis } \\
\text { predominantly in bilateral } \\
\text { ethmoidal air cells }\end{array}$ & $\begin{array}{c}\text { maxillary } \\
\text { fungal } \\
\text { sinusitis }\end{array}$ & $\begin{array}{c}\text { fungal sinusitis with extension in } \\
\text { of cellulitis in posterior aspect of left orbit }\end{array}$ \\
\hline Radiology & &
\end{tabular}

\section{Discussion}

COVID-19 is a spectrum of various pathophysiologic features. Complex interplay of various factors like preexisting diseases, use of immunosuppressive therapy, the risk of hospital-acquired infections, and systemic immune variations may lead to secondary infections. These are increasingly being recognized in view of their impact on morbidity and mortality. ${ }^{1}$ The associated immune dysregulation, with reduced numbers of $T$ lymphocytes, $\mathrm{CD} 4+\mathrm{T}$, and $\mathrm{CD} 8+\mathrm{T}$ cells, may alter innate immunity. ${ }^{2}$ In a recent review, $8 \%$ patients of COVID-19 had secondary bacterial or fungal infections during hospital admission. ${ }^{3}$
Recently a case of young female with mucormycosis and orbital compartment syndrome was reported. ${ }^{4}$ She had no any preexisting disorder except coronavirus disease. Another case of a 60 year old diabetic male was reported with Rhino-Orbital Mucormycosis developed during the course of COVID-19 treatment. ${ }^{5}$

Mucormycosis is a rare fungal infection mainly developing in compromised hosts, and associated with high mortality. ${ }^{6}$ The mucormycosis infection commencing in the nose, causes a rapid gangrene of the nasal mucosa. It may also cause darkening and gangrene of the overlying skin and the hard palate. It may further spread to the eyes and to 
the brain. The spread is generally along the blood vessels as the fungus has a specific predilection for the blood vessel lining and spreads along it. COVID-19 has been noted to cause wide fluctuations in blood sugar levels and may precipitate in uncontrolled diabetes. It may also lead to an increased tendency of clot formation in the blood vessels of the brain and eyes. It is also associated with a decrease in the lymphocyte count and may compromise cell mediated immunity. All these mechanisms seem to be contributing to immune compromise and leading to the increasing incidence of mucormycosis in these patients. 5

The first case of young diabetic female with poor glycemic control had invasive fungal sinusitis involving bilateral ethmoidal sinuses. Detection of SARS CoV2 IgG antibodies attributes to underlying COVID pathogenesis. Second case of diabetic male with good glycemic control but with previous history of COVID-19 disease developed maxillary fugal sinusitis. The third case had poor glycemic control as well as previous infection with Coronavirus disease. As the pathophysiology of various COVID associated diseases are under constant research we aim to add our experience to the literature. The underlying association of COVID-19 disease and various forms of fungal infections need further evaluation.

\section{Conclusion}

COVID-19 is associated with a significant increase in incidence of secondary infections, probably due to immune dysregulation. The widespread use of steroids/ monoclonal antibodies/ broad-spectrum antibiotics as part of COVID-19 treatment may lead to exacerbation of preexisting fungal diseases. It is necessary to consider the possibility of invasive secondary fungal infections in patients with COVID-19 infection especially in those with preexisting risk factors for early diagnosis and treatment with the subsequent reduction of mortality and morbidity.

\section{Conflict of Interest: None}

\section{References}

1. Chen N, Zhou M, Dong X, Qu J, Gong F, Han Y et al. Epidemiological and clinical characteristics of 99 cases of 2019 novel coronavirus pneumonia in Wuhan, China: a descriptive study. Lancet 2020.

2. Gangneux JP, Bougnoux ME, Dannaoui E, Cornet $M$, Zahar JR. Invasive fungal diseases during COVID-19: We should be prepared. Journal de Mycologie Medicale 2020.

3. Rawson TM, Moore LSP, Zhu N, Ranganathan N, Skolimowska K, Gilchrist M et al. Bacterial and Fungal Coinfection in Individuals With Coronavirus: A Rapid Review To Support COVID-19 Antimicrobial Prescribing. Clinical infectious diseases: an official publication of the Infectious Diseases Society of America. 2020.
4. Werthman-Ehrenreich A. Mucormycosis with orbital compartment syndrome in a patient with COVID-19. Am J Emerg Med 2020.

5. Mehta S, Pandey A. Rhino-Orbital Mucormycosis Associated With COVID-19. Cureus 2020.

6. Kudo K, Hasegawa H, Sato E, Kaneko T, Ishida D, Kanno Cet al. A case of rhinocerebral mucormycosis extending into the skull. J Oral Maxillofac Surgery, Med Pathol 2017. 